\title{
Weighted Fusion Robust Steady-State Kalman Filters for Multisensor System with Uncertain Noise Variances
}

\author{
Wen-Juan Qi, Peng Zhang, and Zi-Li Deng \\ Department of Automation, Heilongjiang University, Harbin 150080, China \\ Correspondence should be addressed to Zi-Li Deng; dzl@hlju.edu.cn
}

Received 18 November 2013; Accepted 7 March 2014; Published 30 April 2014

Academic Editor: Jong Hae Kim

Copyright ( 2014 Wen-Juan Qi et al. This is an open access article distributed under the Creative Commons Attribution License, which permits unrestricted use, distribution, and reproduction in any medium, provided the original work is properly cited.

\begin{abstract}
A direct approach of designing weighted fusion robust steady-state Kalman filters with uncertain noise variances is presented. Based on the steady-state Kalman filtering theory, using the minimax robust estimation principle and the unbiased linear minimum variance (ULMV) optimal estimation rule, the six robust weighted fusion steady-state Kalman filters are designed based on the worst-case conservative system with the conservative upper bounds of noise variances. The actual filtering error variances of each fuser are guaranteed to have a minimal upper bound for all admissible uncertainties of noise variances. A Lyapunov equation method for robustness analysis is proposed. Their robust accuracy relations are proved. A simulation example verifies their robustness and accuracy relations.
\end{abstract}

\section{Introduction}

The multisensor information fusion (multisource information fusion or multisensor data fusion) has been applied widely in many fields including guidance, defense, robotics, target tracking, signal processing, GPS positioning, unmanned aerial vehicle (UAV), communication, command, control, computer, and intelligent systems $\left(\mathrm{C}^{4} \mathrm{I}\right)$ and has attracted significant interest in recent years. Over the past two decades, many fused Kalman filtering algorithms have been developed to handle the state and signal estimation problems for the multisensor systems. The aim is how to combine the local estimators or local measurements obtained from each sensor to obtain a fused estimator, whose accuracy is higher than that of each local estimator [1]. The basic fused filtering algorithms include the centralized and distributed algorithms depending on whether the measurements' information is directly communicated to the fusion center or not [2]. For the centralized fusion algorithm, all the measurement data from local sensor are carried to the fusion center which can give the globally optimal fused state estimation, but its disadvantage is requiring a larger computation and communication burden. The distributed fusion algorithms can give the globally optimal or suboptimal state estimation by combing or weighting the local state estimators, whose advantages are that they can reduce the communication burden, and is more robust and reliable, and also has stronger fault tolerance. Under the ULMV rule, there are three optimal distributed fusion algorithms weighted by matrices, diagonal matrices, and scalars, respectively [3-5]. The optimal weighted measurement fusion algorithms can give the global optimal state estimation by weighting the local measurements to obtain a fusion measurement equation, accompanied with the state equation; based on a single Kalman filter, two optimal weighted measurements fusion algorithms were presented in [6-8].

The classical Kalman filtering is only suitable to handle the state estimation problems for the systems that the model parameters and noises variances are precisely known. However, in many application problems, the uncertainties of model parameters and noise variances are widely found. In the presence of uncertainties, the filter performance is degraded and may yield the filter divergence. In order to solve the filtering problems for uncertain systems, in recent years, several results have been derived on the design of robust Kalman filters. The so-called robust Kalman filtering is to design a filter to guarantee a minimal upper bound of the actual filtering error variances for all admissible uncertainties. There are two basic approaches to solve this problem, which are the Riccati equation approach [9-15] and 
the linear matrix inequality (LMI) approach [16-20]. These two methods have been applied to design the robust Kalman filter for uncertain systems with the uncertainties of model parameters [9-20], where the noise variances are assumed to be exactly known. However, the design of the robust Kalman filters with uncertain noise variances is seldom reported [2124].

Several robust Kalman filters only consider the stochastic systems with single sensor, while the design of the multisensor information fusion robust Kalman filters is seldom considered and the robustness of the fused Kalman filters was not proved [25-27].

The robust Kalman filters design includes the finitehorizon (time-varying) robust Kalman filters design and the infinite-horizon (steady-state) robust Kalman filters design. The steady-state robust Kalman filters can be designed by taking the limits for the time-varying robust Kalman filters $[9,10,14]$, and this is called the indirect design method. However, it is seldom reported that applying the steady-state Kalman filtering theory can directly design the steady-state robust Kalman filters, and this is called the direct design method.

For the systems with uncertain model parameters and/or noise variances, the covariance intersection (CI) fusion robust filtering method was presented in [28-31]. Its basic principle is that the robust CI fusion filter can be obtained by the convex combination of the robust local filters. Its advantage is that the cross-covariance of the local filtering errors is avoided, and it is suitable to handle the systems with unknown cross-covariance. Its disadvantage is that the local robust filters are assumed to be known, and the upper bound of the actual fused estimates has larger conservativeness because the information of cross-covariance is ignored. The geometric principle of the CI fusion is that the variance ellipse of the upper bound of actual fusion estimation error variance tightly encloses the intersection region of all variances ellipses of the upper bounds of actual local estimation error variances [32]. Recently, the ellipsoidal intersection (EI) fusion method with the cross-covariance information was presented in [33], which improves the robust accuracy of the CI fusion estimate. The comparison of the CI fusion method with several weighted fusion methods was given in [5]. The CI fusion method has been applied to many fields including remote sensing [34], simultaneous localization and mapping (SLAM) [35], rocket tracking and prediction [36], and vehicle localization [37].

Recently, the robust weighted fusion Kalman filters for multisensor time-varying systems with uncertain noise variances were presented by our team in [24], where the Lyapunov equation method of designing robust Kalman filters, the five robust weighted fusion time-varying Kalman filters based on the minimax robust estimation principle, and the concept of robust accuracy have been presented. By taking the limits for the time-varying robust weighted fusion Kalman filters, the corresponding robust steady-state Kalman filters have been also presented. However, based on the steady-state Kalman filtering theory, the problem to design directly the robust steady-state Kalman filters is not solved, and, based on the cross-covariance information, the problem to reduce the conservativeness of the upper bound of the CI fusion estimate is not solved. In addition, only one robust weighted measurement fuser was presented in [24].

In this paper, based on the steady-state Kalman filtering theory $[38,39]$, for the multisensor time-invariant system with noise variances uncertainties, using the minimax robust estimation principle, for the worst-case conservative system with the conservative upper bound of noise variances, three weighted state fusion robust steady-state Kalman filters will be presented. In order to improve the robust accuracy of the CI fuser and to reduce its conservativeness, a modified robust $\mathrm{CI}$ fuser with the cross-covariance information will be presented. In addition, two weighted measurement fusion robust steady-state Kalman filters will be presented. The proposed robust weighted fusion steady-state Kalman filtering approach is different from that in [24]. Our approach avoids finding the time-varying robust weighted fusers and their limits.

Finally, the robustness of the local and weighting fused robust Kalman filters is proved based on the Lyapunov equation method, which is completely different from the Riccati equation method and LMI method [9-20]. Their robust accuracy relations are strictly proved. In order to verify the correctness of theoretical accuracy relations, a MonteCarlo simulation example for a three-sensor tracking system with uncertain noise variances is given.

The remainder of the paper is organized as follows. The problem formulation is given in Section 2. The local robust steady-state Kalman filters and their robustness analysis are presented in Section 3. Six weighted fusion robust steady-state Kalman filters and their robustness analysis are proposed in Section 4. The comparison of the robust accuracies of the local and fused robust Kalman filters is given in Section 5. Section 6 gives a simulation example. The conclusions are presented in Section 7.

\section{Problem Formulation}

Consider the multisensor time-invariant system with uncertain noise variances

$$
\begin{gathered}
x(t+1)=\Phi x(t)+\Gamma w(t), \\
y_{i}(t)=H_{i} x(t)+v_{i}(t), \quad i=1, \ldots, L,
\end{gathered}
$$

where $t$ is the discrete time, $x(t) \in R^{n}$ is the state to be estimated, $L$ is the number of sensors, $y_{i}(t) \in R^{m_{i}}$ and $v_{i}(t) \epsilon$ $R^{m_{i}}$ are the measurement and measurement noise of the $i$ th subsystem, and $w(t) \in R^{r}$ is the input noise. $\Phi, \Gamma$, and $H_{i}$ are known constant matrices with appropriate dimensions.

Assumption 1. $w(t)$ and $v_{i}(t)$ are uncorrelated white noises with zero mean and unknown uncertain actual variances $\bar{Q}$ and $\bar{R}_{i}$, and $Q$ and $R_{i}$ are known conservative upper bounds of $\bar{Q}$ and $\bar{R}_{i}$, respectively; that is,

$$
\bar{Q} \leq Q, \quad \bar{R}_{i} \leq R_{i}, \quad i=1, \ldots, L,
$$

in the sense that $A \leq B$ means that $B-A \geq 0$ is a positive semidefinite matrix. 
Assumption 2. $(\Phi, \Gamma)$ is a completely controllable pair and $\left(\Phi, H_{i}\right)$ is a completely observable pair.

The problem is to design the local or fused robust steadystate Kalman filter $\widehat{x}(t \mid t)$ such that the variances of actual filtering errors are guaranteed to have a minimal upper bound $S$ for all admissible uncertain noise variances $\bar{Q}$ and $\bar{R}_{i}$ satisfying (3); that is, the actual filtering error variance satisfies

$$
E\left[(x(t)-\widehat{x}(t \mid t))(x(t)-\widehat{x}(t \mid t))^{T}\right] \leq S,
$$

where $E$ is the mathematical expectation operator and the superscript $T$ is the transpose.

Definition 3. The measurements $y_{i}(t)$ generated from the systems (1) and (2) with unknown actual noise variances $\bar{Q}$ and $\bar{R}_{i}, i=1, \ldots, L$, are called the actual measurements $y_{i}(t)$, which are obtained via the sensors, and are available (known).

Definition 4. The measurements $y_{i}(t)$ generated from the systems (1) and (2) with the conservative upper bounds $Q$ and $R_{i}$ of noise variances are called the conservative measurement which are unavailable (unknown).

Definition 5. The Kalman filters with conservative measurements $y_{i}(t)$ are called the conservative Kalman filter which is unrealizable. The Kalman filters with the actual measurements $y_{i}(t)$ are called the actual Kalman filters.

\section{Local Robust Steady-State Kalman Filters}

According to the minimax robust optimal estimation principle [40], consider the worst-case conservative systems (1) and (2) with Assumptions 1-2 and with the conservative upper bounds $Q$ and $R_{i}$ of noise variances; the conservative local steady-state Kalman filters are given as [38, 39]

$$
\begin{aligned}
& \widehat{x}_{i}(t \mid t)=\Psi_{i} \widehat{x}_{i}(t-1 \mid t-1)+K_{i} y_{i}(t), \quad i=1, \ldots, L, \\
& \Psi_{i}=\left[I_{n}-K_{i} H_{i}\right] \Phi, \quad K_{i}=\Sigma_{i} H_{i}^{T}\left(H_{i} \Sigma_{i} H_{i}^{T}+R_{i}\right)^{-1},
\end{aligned}
$$

where $I_{n}$ is an $n \times n$ identity matrix, $\Psi_{i}$ is a stable matrix, and $K_{i}$ is the steady-state filtering gain matrix. Here, the measurements $y_{i}(t)$ are unavailable as in Definition 4.

The conservative one-step prediction error variances $\Sigma_{i}$ satisfy the Riccati equations

$$
\Sigma_{i}=\Phi\left[\Sigma_{i}-\Sigma_{i} H_{i}^{T}\left(H_{i} \Sigma_{i} H_{i}^{T}+R_{i}\right)^{-1} H_{i} \Sigma_{i}\right] \Phi^{T}+\Gamma Q \Gamma^{T} .
$$

The conservative local steady-state filtering error variances satisfy the Lyapunov equations

$$
P_{i}=\Psi_{i} P_{i} \Psi_{i}^{T}+\left[I_{n}-K_{i} H_{i}\right] \Gamma Q \Gamma^{T}\left[I_{n}-K_{i} H_{i}\right]^{T}+K_{i} R_{i} K_{i}^{T},
$$

and the conservative local steady-state filtering error crosscovariances also satisfy the Lyapunov equations [4]

$$
P_{i j}=\Psi_{i} P_{i j} \Psi_{j}^{T}+\left[I_{n}-K_{j} H_{j}\right] \Gamma Q \Gamma^{T}\left[I_{n}-K_{j} H_{j}\right]^{T} .
$$

Notice that the conservative local Kalman filters (5) are unrealizable, because the conservative measurements $y_{i}(t)$ given in Definition 4 are unavailable. Only the actual measurements $y_{i}(t)$ measured via sensors are available, which are generated from systems (1) and (2) with the actual noise variances $\bar{Q}$ and $\bar{R}_{i}, i=1, \ldots, L$. Therefore, replacing the conservative measurements $y_{i}(t)$ with the actual measurements $y_{i}(t)$ in (5), we obtain the actual local Kalman filters $\widehat{x}_{i}(t \mid t)$.

Define the actual local steady-state filtering error variance as

$$
\bar{P}_{i}=E\left[\tilde{x}_{i}(t \mid t) \tilde{x}_{i}^{\mathrm{T}}(t \mid t)\right], \quad \tilde{x}_{i}(t \mid t)=x(t)-\widehat{x}_{i}(t \mid t) .
$$

Substituting (1) and (5) into $\widetilde{x}_{i}(t \mid t)=x(t)-\widehat{x}_{i}(t \mid t)$, we obtain that

$$
\begin{aligned}
\widetilde{x}_{i}(t \mid t)= & \Phi x(t-1)+\Gamma w(t-1) \\
& -\Psi_{i} \widehat{x}(t-1 \mid t-1)-K_{i} y_{i}(t) .
\end{aligned}
$$

Substituting the actual measurements (2) into (11) yields

$$
\begin{aligned}
\tilde{x}_{i}(t \mid t)= & \Psi_{i} \tilde{x}(t-1 \mid t-1) \\
& +\left(I_{n}-K_{i} H_{i}\right) \Gamma w(t-1)-K_{i} v_{i}(t) .
\end{aligned}
$$

Substituting (12) into (10) yields the actual steady-state filtering error variances as

$$
\bar{P}_{i}=\Psi_{i} \bar{P}_{i} \Psi_{i}^{T}+\left[I_{n}-K_{i} H_{i}\right] \Gamma \bar{Q} \Gamma^{T}\left[I_{n}-K_{i} H_{i}\right]^{T}+K_{i} \bar{R}_{i} K_{i}^{T} .
$$

Applying (12), the actual steady-state filtering error crosscovariances are obtained as

$$
\bar{P}_{i j}=\Psi_{i} \bar{P}_{i j} \Psi_{j}^{T}+\left[I_{n}-K_{i} H_{i}\right] \Gamma \bar{Q} \Gamma^{T}\left[I_{n}-K_{j} H_{j}\right]^{T}, \quad i \neq j .
$$

Lemma 6 (see [38]). Consider the Lyapunov equation with $U$ being a symmetric matrix,

$$
P=\Psi P \Psi^{T}+U
$$

If $\Psi$ is a stable matrix (all its eigenvalues are inside the unit circle) and $U \geq 0$, then $P$ is unique and symmetric and $P \geq 0$.

Theorem 7. Formultisensor uncertainsystems (1) and (2) with Assumptions 1-2, the actual local Kalman filters (5) with conservative upper bounds $Q$ and $R_{i}$ of noise variances are robust in the sense that, for all admissible actual variances $\bar{Q}$ and $\bar{R}_{i}$ satisfying (3), one has

$$
\bar{P}_{i} \leq P_{i}, \quad i=1, \ldots, L,
$$

and they are called the local robust steady-state Kalman filters, and $P_{i}$ is the minimal upper bound of $\bar{P}_{i}$.

Proof. Defining $\Delta P_{i}=P_{i}-\bar{P}_{i}$, subtracting (13) from (8) yields the Lyapunov equation

$$
\Delta P_{i}=\Psi_{i} \Delta P_{i} \Psi_{i}^{T}+U_{i},
$$


where

$$
\begin{aligned}
U_{i}= & {\left[I_{n}-K_{i} H_{i}\right] \Gamma(Q-\bar{Q}) \Gamma^{T}\left[I_{n}-K_{i} H_{i}\right]^{T} } \\
& +K_{i}\left(R_{i}-\bar{R}_{i}\right) K_{i}^{T} .
\end{aligned}
$$

Applying (3) and (18) yields $U_{i} \geq 0$, and noting that $\Psi_{i}$ is a stable matrix and applying Lemma 6 , we have $\Delta P_{i} \geq 0$; that is $\bar{P}_{i} \leq P_{i}$ holds. Taking $\bar{Q}=Q, \bar{R}_{i}=R_{i}$, then the constraints (3) are satisfied, and $U_{i}=0$. Applying Lemma 6 to (17) yields $\Delta P_{i}=0$; that is, $P_{i}=\bar{P}_{i}$. For arbitrary other upper bound $P_{i}^{*}$, we have $P_{i}=\bar{P}_{i} \leq P_{i}^{*}$, which yields that $P_{i}$ is the minimal upper bound of $\bar{P}_{i}$. The proof is completed.

\section{Weighted Fusion Robust Steady-State Kalman Filters}

4.1. Four Robust Weighted State Fusion Steady-State Kalman Filters. For the worst-case conservative multisensor systems (1) and (2) with Assumptions 1-2, and with conservative upper bounds $Q$ and $R_{i}$, under the ULMV fusion rule, the four conservative steady-state optimal weighted fusion Kalman filters are given by [3-5]

$$
\widehat{x}_{\theta}(t \mid t)=\sum_{i=1}^{L} \Omega_{i}^{\theta} \widehat{x}_{i}(t \mid t), \quad \theta=m, s, d, \mathrm{CI}
$$

with the constraint of unbiasedness

$$
\sum_{i=1}^{L} \Omega_{i}^{\theta}=I_{n}
$$

where $\theta=m, s, d$ and CI denote the fusers weighted by matrices, scalars, diagonal matrices, and the CI fuser, respectively.

The optimal weighted matrices are computed as [3-5]

$$
\begin{gathered}
{\left[\Omega_{1}^{m} \cdots \Omega_{L}^{m}\right]=\left(e^{T} P^{-1} e\right)^{-1} e^{T} P^{-1}, \quad e=\left[I_{n} \cdots I_{n}\right]^{T},} \\
P=\left(P_{i j}\right)_{n L \times n L}
\end{gathered}
$$

with the definition $P_{i i}=P_{i}$.

The conservative fused filtering error variance is given as

$$
P_{m}=\left(e^{T} P^{-1} e\right)^{-1} .
$$

The optimal scalars weights are computed as

$$
\begin{gathered}
{\left[\omega_{1}, \ldots, \omega_{L}\right]=\left(e^{T} P_{\operatorname{tr}}^{-1} e\right)^{-1} e^{T} P_{\text {tr }}^{-1},} \\
\Omega_{i}^{s}=\omega_{i} I_{n}, \quad i=1, \ldots, L,
\end{gathered}
$$

where $e=[1 \cdots 1]^{T}$ and the $L \times L$ matrix $P_{\mathrm{tr}}(t \mid t)$ is defined as

$$
P_{\mathrm{tr}}=\left(\operatorname{tr} P_{i j}\right)_{L \times L}
$$

where $\operatorname{tr} P_{i j}$ denotes the trace of $P_{i j}$. The conservative fused error variance is given as

$$
P_{s}=\sum_{i=1}^{L} \sum_{j=1}^{L} \omega_{i} \omega_{j} P_{i j}
$$

The optimal diagonal matrix weights are computed as

$$
\begin{gathered}
\Omega_{i}^{d}=\operatorname{diag}\left(\omega_{i 1}, \ldots, \omega_{i n}\right), \\
{\left[\omega_{1 i} \cdots \omega_{L i}\right]=\left(e^{T}\left(P^{i i}\right)^{-1} e\right)^{-1} e^{T}\left(P^{i i}\right)^{-1}, \quad i=1, \ldots, n,} \\
e=[1 \cdots 1]^{T}, \quad P^{i i}=\left(P_{s k}^{i i}\right)_{L \times L^{\prime}}
\end{gathered}
$$

where $P_{s k}^{i i}$ is the $(i, i)$ th diagonal element of $P_{s k}, s, k=1, \ldots, L$.

The conservative fused error variance is given as

$$
P_{d}=\sum_{i=1}^{L} \sum_{j=1}^{L} \Omega_{i}^{d} P_{i j} \Omega_{j}^{d T} .
$$

The CI fusion weights are computed as $[5,31,32]$

$$
\begin{gathered}
\Omega_{\mathrm{CI}}=\left[\Omega_{1}^{\mathrm{CI}}, \ldots, \Omega_{\mathrm{L}}^{\mathrm{CI}}\right], \quad \Omega_{i}^{\mathrm{CI}}=\omega_{i} P_{\mathrm{CI}}^{*} P_{i}^{-1}, \quad i=1, \ldots, L, \\
P_{\mathrm{CI}}^{*}=\left[\sum_{i=1}^{L} \omega_{i} P_{i}^{-1}\right]^{-1} .
\end{gathered}
$$

The optimal weighting coefficients $\omega_{i}$ are obtained by minimizing the performance index

$$
\min _{\omega_{i}} \operatorname{tr} P_{\mathrm{CI}}^{*}=\min _{\substack{\omega_{i} \in[0,1] \\ \omega_{1}+\cdots+\omega_{L}=1}} \operatorname{tr}\left\{\left[\sum_{i=1}^{L} \omega_{i} P_{i}^{-1}\right]^{-1}\right\} .
$$

This needs to solve L-dimension nonlinear convex optimization problem, which can be solved by "fmincon" function in MATLAB toolbox.

Define

$$
\Omega_{\theta}=\left[\Omega_{1}^{\theta}, \ldots, \Omega_{L}^{\theta}\right], \quad \theta=m, s, d, \text { CI } .
$$

From (20), we have

$$
x(t)=\sum_{i=1}^{L} \Omega_{i}^{\theta} x(t), \quad \theta=m, s, d, \mathrm{CI} .
$$

Subtracting (19) from (34) yields the conservative fused filtering errors as

$$
\tilde{x}_{\theta}(t \mid t)=\sum_{i=1}^{L} \Omega_{i}^{\theta} \tilde{x}_{i}(t \mid t), \quad \theta=m, s, d, \text { CI } .
$$

Applying (34) and (35) yields the conservative fused filtering error variances having a unified form

$$
P_{\theta}=\Omega_{\theta} P \Omega_{\theta}^{T}, \quad \theta=m, s, d, \mathrm{CI}
$$


with $P$ defined in (22). Replacing the conservative local Kalman filters $\hat{x}_{i}(t \mid t)$ in (19) by the actual local Kalman filters $\widehat{x}_{i}(t \mid t)$, we obtain the actual weighted fusion Kalman filters.

Define the actual weighted fusion filtering error variance $\bar{P}_{\theta}(t \mid t)$ as

$$
\bar{P}_{\theta}(t \mid t)=E\left[\tilde{x}_{\theta}(t \mid t) \tilde{x}_{\theta}^{\mathrm{T}}(t \mid t)\right], \quad \theta=m, s, d, \mathrm{CI},
$$

where $\tilde{x}_{\theta}(t \mid t)=x(t)-\widehat{x}_{\theta}(t \mid t)$ and $\hat{x}_{\theta}(t \mid t)$ is the actual fused filters (19) with $\hat{x}_{i}(t \mid t)(i=1, \ldots, L)$ being the actual local Kalman filters. From (35) and (37), we obtain the actual fused filtering error variances as

$$
\bar{P}_{\theta}=\Omega_{\theta} \bar{P} \Omega_{\theta}^{T}, \quad \theta=m, s, d, \mathrm{CI},
$$

with the definition

$$
\bar{P}=\left(\bar{P}_{i j}\right)_{n L \times n L}
$$

In particular, from (30), (36), and (38), taking $\theta=\mathrm{CI}$, the CI fuser has the conservative and actual steady-state fused error variances as

$$
\begin{aligned}
& P_{\mathrm{CI}}=P_{\mathrm{CI}}^{*}\left[\sum_{i=1}^{L} \sum_{j=1}^{L} \omega_{i} P_{i}^{-1} P_{i j} P_{j}^{-1} \omega_{j}\right] P_{\mathrm{CI}}^{*} \\
& \bar{P}_{\mathrm{CI}}=P_{\mathrm{CI}}^{*}\left[\sum_{i=1}^{L} \sum_{j=1}^{L} \omega_{i} P_{i}^{-1} \bar{P}_{i j} P_{j}^{-1} \omega_{j}\right] P_{\mathrm{CI}}^{*}
\end{aligned}
$$

Notice that $P_{\mathrm{CI}}$ is defined with the conservative crosscovariance $P_{i j}$.

Lemma 8 (see [24]). Let $\Lambda$ be the $r \times r$ positive semidefinite matrix; that is, $\Lambda \geq 0$; then the following $r L \times r L$ matrix $\Lambda_{\delta}$ is also positive semidefinite; that is,

$$
\Lambda_{\delta}=\left[\begin{array}{ccc}
\Lambda & \cdots & \Lambda \\
\vdots & \ddots & \vdots \\
\Lambda & \cdots & \Lambda
\end{array}\right]_{r L \times r L} \geq 0
$$

Lemma 9 (see [24]). Let $R_{i}$ be the $m_{i} \times m_{i}$ positive semidefinite matrix; that is, $R_{i} \geq 0$; the following $m \times m$ block-diagonal matrix $R_{\delta}$ is also positive semidefinite; that is,

$$
R_{\delta}=\operatorname{diag}\left(R_{1}, \ldots, R_{L}\right) \geq 0
$$

with $m=m_{1}+\cdots+m_{L}$.

Theorem 10. For multisensor uncertain systems (1) and (2) with Assumptions 1-2 and with conservative upper bounds $Q$ and $R_{i}$ of noise variances, the actual four steady-state weighted Kalman fusers are robust in the sense that, for all admissible actual variances $\bar{Q}$ and $\bar{R}_{i}$ satisfying (3), one has

$$
\bar{P}_{\theta} \leq P_{\theta}, \quad \theta=m, s, d, C I,
$$

and they are called the robust weighted fusion steady-state Kalman filters, and $P_{\theta}$ is the minimal upper bound of $\bar{P}_{\theta}$.
Proof. Defining $\Delta P_{\theta}=P_{\theta}-\bar{P}_{\theta}$, subtracting (36) from (38) yields

$$
\Delta P_{\theta}=\Omega_{\theta}(P-\bar{P}) \Omega_{\theta}^{T}
$$

In order to prove the robustness $\Delta P_{\theta}=P_{\theta}-\bar{P}_{\theta} \geq 0$, we only need to prove that the inequality $P-\bar{P} \geq 0$ holds.

Applying (8) and (9) yields the following Lyapunov equation

$$
P=\Psi P \Psi^{T}+U Q_{a} U^{T}+K R K^{T}
$$

where we define

$$
\begin{aligned}
\Psi & =\left[\begin{array}{lll}
\Psi_{1} & & 0 \\
& \ddots & \\
0 & & \Psi_{L}
\end{array}\right], \\
U & =\left[\begin{array}{ccc}
\left(I_{n}-K_{1} H_{1}\right) \Gamma & \ddots \\
& 0 & \\
& 0 & \left(I_{n}-K_{L} H_{L}\right) \Gamma
\end{array}\right], \\
Q_{a} & =\left[\begin{array}{lll}
Q & & Q \\
Q & \ddots & Q
\end{array}\right], \\
K & =\left[\begin{array}{lll}
K_{1} & & 0 \\
0 & \ddots & K_{L}
\end{array}\right], \\
R & =\left[\begin{array}{lll}
R_{1} & & 0 \\
0 & \ddots & R_{L}
\end{array}\right] .
\end{aligned}
$$

Similarly, applying (13) and (14), $\bar{P}$ can be expressed as

$$
\bar{P}=\Psi \bar{P} \Psi^{T}+U \bar{Q}_{a} U^{T}+K \bar{R} K^{T} .
$$

with the definitions

$$
\bar{Q}_{a}=\left[\begin{array}{ccc}
\bar{Q} & & \bar{Q} \\
\vdots & \ddots & \vdots \\
\bar{Q} & & \bar{Q}
\end{array}\right], \quad \bar{R}=\left[\begin{array}{ccc}
\bar{R}_{1} & & 0 \\
& \ddots & \\
0 & & \bar{R}_{L}
\end{array}\right]
$$

Since $\Psi_{i}$ is a stable matrix, then the eigenvalues of the matrix $\Psi_{i}$ are all within the unit circle and are determined from its characteristic equation $\operatorname{det}\left(\lambda I_{n}-\Psi_{i}\right)=0$. The eigenvalues of the matrix $\Psi$ are determined from the characteristic equation

$$
\operatorname{det}\left(\lambda I_{n L}-\Psi\right)=\operatorname{det}\left(\lambda I_{n}-\Psi_{1}\right) \cdots \operatorname{det}\left(\lambda I_{n}-\Psi_{L}\right)=0 \text {, }
$$

which yields that $\Psi$ is also a stable matrix, because the eigenvalues of $\Psi_{i}$ are also the eigenvalues of $\Psi$.

Denoting $\Delta P=P-\bar{P}$, subtracting (48) from (46) yields the Lyapunov equation

$$
\Delta P=\Psi \Delta P \Psi^{T}+U\left(Q_{a}-\bar{Q}_{a}\right) U^{T}+K(R-\bar{R}) K^{T} .
$$

Noting that $\bar{Q} \leq Q, \bar{R}_{i} \leq R_{i}$, applying Lemmas 8 and 9 yields $\bar{Q}_{a} \leq Q_{a}, \bar{R} \leq R$; therefore $U\left(Q_{a}-\bar{Q}_{a}\right) U^{T}+K(R-$ 
$\bar{R}) K^{T} \geq 0$. Noting that $\Psi$ is a stable matrix and applying Lemma 6 to (51), we have $\Delta P \geq 0$; that is,

$$
\bar{P} \leq P .
$$

From (45) and (52), we have $\Delta P_{\theta} \geq 0$, so (44) holds. If $P_{\theta}^{*}$ is other upper bound of $\bar{P}_{\theta}$, taking $\bar{Q}=Q, \bar{R}_{i}=R_{i}$, we have $\bar{Q}_{a}=Q_{a}, \bar{R}=R$, so applying Lemma 6 to (51) yields $\Delta P=0$; that is, $\bar{P}=P$. Hence, applying (45) yields $\Delta P_{\theta}=0$, so $P_{\theta}=\bar{P}_{\theta} \leq P_{\theta}^{*}$, which yields that $P_{\theta}$ is the minimal upper bound of $\bar{P}_{\theta}$. The proof is completed.

Remark 11. From (19) and (30), the CI fusion Kalman filter can be rewritten as

$$
\widehat{x}_{\mathrm{CI}}(t \mid t)=P_{\mathrm{CI}}^{*} \sum_{i=1}^{L} \omega_{i} P_{i}^{-1} \widehat{x}_{i}(t \mid t),
$$

which can be reviewed as a special fuser weighted by matrices with weights $\Omega_{i}^{\mathrm{CI}}=\omega_{i} P_{\mathrm{CI}}^{*} P_{i}^{-1}$.

From (31) and (53), the CI fuser has the convex combination form as

$$
\begin{aligned}
\left(P_{\mathrm{CI}}^{*}\right)^{-1} \widehat{x}_{\mathrm{CI}}(t \mid t) & =\sum_{i=1}^{L} \omega_{i} P_{i}^{-1} \widehat{x}_{i}(t \mid t), \\
\left(P_{\mathrm{CI}}^{*}\right)^{-1} & =\sum_{i=1}^{L} \omega_{i} P_{i}^{-1}
\end{aligned}
$$

and it is proved [5] that $P_{\mathrm{CI}}^{*}$ is a conservative upper bound of $\bar{P}_{\mathrm{CI}}$,

$$
\bar{P}_{\mathrm{CI}} \leq P_{\mathrm{CI}}^{*}
$$

From (31) or (55), the upper bound $P_{\mathrm{CI}}^{*}$ is defined without the cross-covariance information and is only determined by the conservative local variances $P_{i}$, so that $P_{\mathrm{CI}}^{*}$ has certain conservativeness; that is, $P_{\mathrm{CI}}^{*}$ is not a minimal upper bound of $\bar{P}_{\mathrm{CI}}$ for all admissible uncertainties of noise variances. From Theorem 10, we have the robustness

$$
\bar{P}_{\mathrm{CI}} \leq P_{\mathrm{CI}}
$$

and $P_{\mathrm{CI}}$ defined by (40) with the conservative crosscovariance $P_{i j}$, is the minimal upper bound of $\bar{P}_{\mathrm{CI}}$. Hence, we have

$$
P_{\mathrm{CI}} \leq P_{\mathrm{Cl}}^{*}
$$

that is, the upper bound $P_{\mathrm{CI}}$ has less conservativeness than $P_{\mathrm{CI}}^{*}$.

4.2. Two Robust Weighted Measurement Fusion Steady-State Kalman Filters. For the worst-case conservative systems (1) and (2) with Assumptions 1-2, and with the conservative upper bounds $Q$ and $R_{i}$ of noise variances, if $H_{i}$ have the common $m \times n$ right factor $H$, that is,

$$
H_{i}=M_{i} H, \quad i=1, \ldots, L,
$$

where $M_{i}$ is $m_{i} \times n$ matrix, and the matrix $M^{(0) T} R^{(0)-1} M^{(0)}$ or $H^{(0) T} R^{(0)-1} H^{(0)}$ is assumed to be invertible, with the definition

$$
M^{(0)}=\left[M_{1}^{T}, \ldots, M_{L}^{T}\right]^{T}, \quad H^{(0)}=\left[H_{1}^{T}, \ldots, H_{L}^{T}\right]^{T},
$$

we have the conservative centralized fusion measurement equation

$$
\begin{aligned}
& y^{(0)}(t)=H^{(0)} x(t)+v^{(0)}(t), \\
& y^{(0)}(t)=\left[y_{1}^{T}(t), \ldots, y_{L}^{T}(t)\right]^{T}, \\
& v^{(0)}(t)=\left[v_{1}^{T}(t), \ldots, v_{L}^{T}(t)\right]^{T},
\end{aligned}
$$

Where, according to Assumption 1 , the fused noise $v^{(0)}(t)$ has the conservative variance matrix

$$
R^{(0)}=\operatorname{diag}\left(R_{1}, \ldots, R_{L}\right) .
$$

If (59) holds, then (61) becomes

$$
y^{(0)}(t)=M^{(0)} H x(t)+v^{(0)}(t) .
$$

If $M^{(0) T} R^{(0)-1} M^{(0)}$ is invertible, applying the weighted least squares (WLS) method [34], $H x(t)$ has the WLS estimate

$$
y^{(1)}(t)=\left[M^{(0) T} R^{(0)-1} M^{(0)}\right]^{-1} M^{(0) T} R^{(0)-1} y^{(0)}(t) .
$$

Substituting (64) into (65) yields the first conservative weighted measurement fusion equation

$$
\begin{gathered}
y^{(1)}(t)=H^{(1)} x(t)+v^{(1)}(t), \quad H^{(1)}=H, \\
v^{(1)}(t)=\left[M^{(0) T} R^{(0)-1} M^{(0)}\right]^{-1} M^{(0) T} R^{(0)-1} v^{(0)}(t),
\end{gathered}
$$

Where, from (67), the fused noise $v^{(1)}(t)$ has the variance matrix

$$
R^{(1)}=\left[M^{(0) T} R^{(0)-1} M^{(0)}\right]^{-1} .
$$

If $H^{(0) T} R^{(0)-1} H^{(0)}$ is invertible, from (61), $x(t)$ has the WLS estimate

$$
y^{(2)}(t)=\left[H^{(0) T} R^{(0)-1} H^{(0)}\right]^{-1} H^{(0) T} R^{(0)-1} y^{(0)}(t) .
$$

Substituting (61) into (69) yields the second conservative measurement fusion equation

$$
\begin{gathered}
y^{(2)}(t)=H^{(2)} x(t)+v^{(2)}(t), \quad H^{(2)}=I_{n}, \\
v^{(2)}(t)=\left[H^{(0) T} R^{(0)-1} H^{(0)}\right]^{-1} H^{(0) T} R^{(0)-1} v^{(0)}(t),
\end{gathered}
$$

where from $(71)$ the fused noise $v^{(2)}(t)$ has the variance matrix

$$
R^{(2)}=\left[H^{(0) T} R^{(0)-1} H^{(0)}\right]^{-1} .
$$

Hence, the centralized fusion system and two measurement fusion systems have a unified form as

$$
\begin{gathered}
x(t+1)=\Phi x(t)+\Gamma w(t), \\
y^{(j)}(t)=H^{(j)} x(t)+v^{(j)}(t), \quad j=0,1,2 .
\end{gathered}
$$


Theorem 12. For the worst-case multisensor uncertain system with Assumptions 1-2, and with the conservative upper bounds $Q$ and $R_{i}$ of noise variances, the robust centralized fusion Kalman filter and two robust weighted measurement fusion Kalman filters have a unified form as

$$
\begin{aligned}
\hat{x}^{(j)}(t \mid t)= & \Psi^{(j)} \widehat{x}^{(j)}(t-1 \mid t-1)+K^{(j)} y^{(j)}(t), \quad j=0,1,2 \\
\Psi^{(j)}= & {\left[I_{n}-K^{(j)} H^{(j)}\right] \Phi, } \\
K^{(j)}= & \Sigma^{(j)} H^{(j) T}\left[H^{(j)} \Sigma^{(j)} H^{(j) T}+R^{(j)}\right]^{-1} \\
\Sigma^{(j)}= & \Phi\left[\Sigma^{(j)}-\Sigma^{(j)} H^{(j) T}\left(H^{(j)} \Sigma^{(j)} H^{(j) T}+R^{(j)}\right)^{-1}\right. \\
& \left.\times H^{(j)} \Sigma^{(j)}\right] \Phi^{T}+\Gamma Q \Gamma^{T},
\end{aligned}
$$

and the conservative and actual fused error variances are, respectively, given as

$$
\begin{aligned}
P^{(j)}= & \Psi^{(j)} P^{(j)} \Psi^{(j) T} \\
& +\left[\mathrm{I}_{n}-K^{(j)} H^{(j)}\right] \Gamma Q \Gamma^{T}\left[I_{n}-K^{(j)} H^{(j)}\right]^{T} \\
& +K^{(j)} R^{(j)} K^{(j) T}, \\
\bar{P}^{(j)}= & \Psi^{(j)} \bar{P}^{(j)} \Psi^{(j) T} \\
& +\left[\mathrm{I}_{n}-K^{(j)} H^{(j)}\right] \Gamma \bar{Q} \Gamma^{T}\left[I_{n}-K^{(j)} H^{(j)}\right]^{T} \\
& +K^{(j)} \bar{R}^{(j)} K^{(j) T} .
\end{aligned}
$$

From (62), (67), and (71), applying Assumption 1 yields

$$
\begin{gathered}
\bar{R}^{(0)}=\operatorname{diag}\left(\bar{R}_{1}, \ldots, \bar{R}_{L}\right), \\
\bar{R}^{(1)}=\left[M^{(0) T} \bar{R}^{(0)-1} M^{(0)}\right]^{-1} M^{(0) T} R^{(0)-1} \\
\times \bar{R}^{(0)-1} R^{(0)-1} M^{(0)}\left[M^{(0) T} \bar{R}^{(0)-1} M^{(0)}\right]^{-1}, \\
\bar{R}^{(2)}=\left[H^{(0) T} \bar{R}^{(0)-1} H^{(0)}\right]^{-1} H^{(0) T} R^{(0)-1} \\
\times \bar{R}^{(0)-1} R^{(0)-1} H^{(0)}\left[H^{(0) T} \bar{R}^{(0)-1} H^{(0)}\right]^{-1} .
\end{gathered}
$$

We have three equivalent robust Kalman filters

$$
\widehat{x}^{(0)}(t \mid t)=\widehat{x}^{(1)}(t \mid t)=\widehat{x}^{(2)}(t \mid t)
$$

with the robustness

$$
\bar{P}^{(j)} \leq P^{(j)}, \quad j=0,1,2,
$$

and we have the accuracy relations

$$
\begin{array}{cc}
P^{(0)}=P^{(1)}=P^{(2)}, & \bar{P}^{(0)}=\bar{P}^{(1)}=\bar{P}^{(2)}, \\
\operatorname{tr} P^{(0)}=\operatorname{tr} P^{(1)}=\operatorname{tr} P^{(2)}, & \operatorname{tr} \bar{P}^{(0)}=\operatorname{tr} \bar{P}^{(1)}=\operatorname{tr} \bar{P}^{(2)} .
\end{array}
$$

Proof. The robust Kalman filters (74) have the equivalent information filter form [38]

$$
\begin{aligned}
\widehat{x}^{(j)}(t \mid t) & =\Psi^{(j)} \widehat{x}^{(j)}(t-1 \mid t-1)+K^{(j)} y^{(j)}(t), \quad j=0,1,2, \\
\Psi^{(j)} & =P^{(j)} \Sigma^{(j)} \Phi \\
K^{(j)} & =P^{(j)} H^{(j) T} R^{(j)-1} \\
\Sigma^{(j)} & =\Phi P^{(j)} \Phi^{T}+\Gamma Q \Gamma^{T} \\
P^{(j)} & =\Sigma^{(j)-1}+H^{(j)} R^{(j)-1} H^{(j) T} .
\end{aligned}
$$

From (81), we see that, in order to prove (77) and (79), we only need to prove

$$
\begin{gathered}
H^{(0) T} R^{(0)-1} H^{(0)}=H^{(1) T} R^{(1)-1} H^{(1)}=H^{(2) T} R^{(2)-1} H^{(2)}, \\
H^{(0) T} R^{(0)-1} y^{(0)}(t)=H^{(1) T} R^{(1)-1} y^{(1)}(t)=H^{(2) T} R^{(2)-1} y^{(2)}(t) .
\end{gathered}
$$

Applying (59), (60), (63), (65), (68), (69), and (72), we easily verify that (82) hold. In order to prove (78), from (79), we only need to prove $\bar{P}^{(0)} \leq P^{(0)}$; that is, the centralized fusion Kalman filter is robust. In fact, applying (3) and Lemma 9 yields $\bar{R}^{(0)} \leq R^{(0)}$, so applying the derivation similar to Theorem 7 yields $\bar{P}^{(0)} \leq P^{(0)}$. The proof is completed.

\section{Robust Accuracy Analysis}

Theorem 13. For multisensor uncertain systems (1) and (2) with Assumptions 1-2, the local and fused Kalman filters have the following accuracy relations:

$$
\begin{gathered}
\bar{P}_{\theta} \leq P_{\theta}, \quad \theta=1, \ldots, L, m, s, d, C I, \\
\bar{P}^{(j)} \leq P^{(j)}, \quad j=0,1,2, \\
P^{(0)}=P^{(1)}=P^{(2)}, \quad \bar{P}^{(0)}=\bar{P}^{(1)}=\bar{P}^{(2)}, \\
P^{(0)} \leq P_{m}, P_{m} \leq P_{\theta}, \quad \theta=1, \ldots, L, m, s, d, \\
P_{C I} \leq P_{C I}^{*}, \quad \bar{P}_{C I} \leq P_{C I}^{*}, \\
\operatorname{tr} \bar{P}_{\theta} \leq \operatorname{tr} P_{\theta}, \quad \theta=1, \ldots, L, m, s, d, C I, \\
\operatorname{tr} \bar{P}^{(j)} \leq \operatorname{tr} P^{(j)}, \quad j=0,1,2,
\end{gathered}
$$

$$
\operatorname{tr} P^{(0)}=\operatorname{tr} P^{(1)}=\operatorname{tr} P^{(2)}, \quad \operatorname{tr} \bar{P}^{(0)}=\operatorname{tr} \bar{P}^{(1)}=\operatorname{tr} \bar{P}^{(2)},
$$

$$
\operatorname{tr} P^{(0)} \leq \operatorname{tr} P_{m}, \quad \operatorname{tr} P_{m} \leq \operatorname{tr} P_{\theta}, \quad \theta=1, \ldots, L, m, s, d,
$$

$$
\operatorname{tr} P_{C I} \leq \operatorname{tr} P_{C I}^{*}, \quad \operatorname{tr} \bar{P}_{C I} \leq \operatorname{tr} P_{C I}^{*},
$$

$$
\operatorname{tr} P_{m} \leq \operatorname{tr} P_{d} \leq \operatorname{tr} P_{s} \leq \operatorname{tr} P_{i}, \quad i=1, \ldots, L .
$$


TABLE 1: The actual and conservative filtering error variances of the local and fused robust Kalman filters.

\begin{tabular}{|c|c|c|c|c|}
\hline$P_{1}$ & $P_{2}$ & $P_{3}$ & $P_{m}$ & $P_{d}$ \\
\hline$\left[\begin{array}{ll}0.2492 & 0.1855\end{array}\right.$ & {$\left[\begin{array}{ll}0.4035 & 0.0645\end{array}\right.$} & {$\left[\begin{array}{ll}0.2087 & 0.1642\end{array}\right.$} & $0.0775 \quad 0.0416$ & {$\left[\begin{array}{ll}0.1039 & 0.0438\end{array}\right.$} \\
\hline$\left[\begin{array}{ll}0.1855 & 0.3046\end{array}\right]$ & $0.0645 \quad 0.121$ & $0.1642 \quad 0.2865$ & 0.04160 .1167 & $0.0438 \quad 0.1173$ \\
\hline $\bar{P}_{1}$ & $\bar{P}_{2}$ & $\bar{P}_{3}$ & $\bar{P}_{m}$ & $\bar{P}_{d}$ \\
\hline$\left[\begin{array}{ll}0.2019 & 0.1497\end{array}\right]$ & {$\left[\begin{array}{ll}0.2922 & 0.0448\end{array}\right.$} & {$\left[\begin{array}{ll}0.1742 & 0.1353\end{array}\right.$} & $0.0607 \quad 0.03$ & {$\left[\begin{array}{ll}0.0828 & 0.0337\end{array}\right.$} \\
\hline$\left[\begin{array}{ll}0.1497 & 0.2447\end{array}\right.$ & $0.0448 \quad 0.0892$ & $0.1353 \quad 0.2327$ & $\begin{array}{ll}0.03 & 0.0878\end{array}$ & $0.0337 \quad 0.0883$ \\
\hline$P_{s}$ & $P_{\mathrm{CI}}^{*}$ & $P_{\mathrm{CI}}$ & $P^{(0)}$ & $P^{(1)}=P^{(2)}$ \\
\hline$\left[\begin{array}{ll}0.1172 & 0.0614\end{array}\right.$ & {$\left[\begin{array}{ll}0.2449 & 0.0882\end{array}\right.$} & $\begin{array}{ll}0.1161 & 0.0487\end{array}$ & $\begin{array}{ll}0.0689 & 0.0414\end{array}$ & {$\left[\begin{array}{ll}0.0689 & 0.0414\end{array}\right.$} \\
\hline$\left[\begin{array}{ll}0.0614 & 0.1554\end{array}\right]$ & 0.08820 .1573 & $0.0487 \quad 0.1199$ & $0.0414 \quad 0.1106$ & $0.0414 \quad 0.1106$ \\
\hline $\bar{P}_{s}$ & $\bar{P}_{\mathrm{CI}}$ & & $\bar{P}^{(0)}$ & $\bar{P}^{(1)}=\bar{P}^{(2)}$ \\
\hline$\left[\begin{array}{ll}0.0896 & 0.0485\end{array}\right]$ & {$\left[\begin{array}{ll}0.0902 & 0.036\end{array}\right.$} & & $0.0537 \quad 0.0304$ & {$\left[\begin{array}{ll}0.0537 & 0.0304\end{array}\right.$} \\
\hline$\left[\begin{array}{ll}0.0485 & 0.1235\end{array}\right]$ & $0.036 \quad 0.0914$ & & $0.0304 \quad 0.0830$ & $0.0304 \quad 0.0830$ \\
\hline
\end{tabular}

Proof. The relations (83)-(85) were proved in Theorems 712. The relation (86) was proved in [5]. The relation (87) was proved in Remark 11. Taking the trace operations for (83)(87) yields (88)-(92). The relation (93) was proved in [5]. The proof is completed.

Remark 14. The trace of the error variance matrix is used to represent the filtering accuracy which is equal to the sum of the filtering error variances for the components of state. The smaller trace means the higher accuracy and the larger trace means the lower accuracy. The accuracy relations (88) and (89) mean that, for any admissible $\bar{Q}$ and $\bar{R}_{i}$ satisfying (3), the actual accuracy $\operatorname{tr} \bar{P}_{\theta}$ or $\operatorname{tr} \bar{P}^{(j)}$ of the local or fused filters $\widehat{x}_{\theta}(t \mid$ t), $\theta=1, \ldots, L, m, d, s, C I, j=0,1,2$, is globally controlled by $\operatorname{tr} P_{\theta}$ or $\operatorname{tr} P^{(j)}$, and $\operatorname{tr} P_{\theta}$ or $\operatorname{tr} P^{(j)}$ is independent of all admissible $\bar{Q}$ and $\bar{R}_{i}$ satisfying (3). Therefore, $\operatorname{tr} P_{\theta}$ or $\operatorname{tr} P^{(j)}$ is called robust accuracy of $\hat{x}_{\theta}(t \mid t)$ and is also called global accuracy [24], and $\operatorname{tr} \bar{P}_{\theta}$ or $\operatorname{tr} \bar{P}^{(j)}$ is called its actual accuracy. Notice that, for different $\bar{Q}$ and $\bar{R}_{i}$, the corresponding actual accuracies are also different; that is, $\operatorname{tr} \bar{P}_{\theta}$ or $\operatorname{tr} \bar{P}^{(j)}$ is related to admissible $\bar{Q}$ and $\bar{R}_{i}$. From (88) and (89), we see that the robust accuracy is the admissible lowest actual accuracy; that is, it is the lowest bound of the actual accuracies. The smaller $\operatorname{tr} P_{\theta}$ or $\operatorname{tr} P^{(j)}$ means the higher robust accuracy, and the larger $\operatorname{tr} P_{\theta}$ or $\operatorname{tr} P^{(j)}$ means the lower robust accuracy.

Remark 15. From Theorem 10, we see that $P_{\mathrm{CI}}$ with the crosscovariance information is a minimal upper bound of $\bar{P}_{\mathrm{CI}}$, and, from (57) and (58), we have the robust accuracy relation

$$
\operatorname{tr} \bar{P}_{\mathrm{CI}} \leq \operatorname{tr} P_{\mathrm{CI}} \leq \operatorname{tr} P_{\mathrm{CI}}^{*}
$$

This means that the robust accuracy of the CI fuser is $\operatorname{tr} P_{\mathrm{CI}}$ rather than $\operatorname{tr} P_{\mathrm{CI}}^{*}$, so that the modified robust accuracy $\operatorname{tr} P_{\mathrm{CI}}$ is higher than the original robust accuracy $\operatorname{tr} P_{\mathrm{CI}}^{*}$ in [24], and it also develops and extends the ellipsoidal intersection (EI) fuser with the cross-covariance information [33].

\section{Simulation Example}

Consider a three-sensor tracking system with uncertain noise variances

$$
\begin{aligned}
x(t+1) & =\Phi x(t)+\Gamma w(t), \\
y_{i}(t) & =H_{i} x(t)+v_{i}(t), \quad i=1,2,3, \\
\Phi & =\left[\begin{array}{cc}
1 & T_{0} \\
0 & 1
\end{array}\right], \quad \Gamma=\left[\begin{array}{c}
0.5 T_{0}^{2} \\
T_{0}
\end{array}\right], \\
H_{1} & =\left[\begin{array}{ll}
1 & 0
\end{array}\right], \quad H_{2}=I_{2}, \quad H_{3}=\left[\begin{array}{ll}
1 & 0
\end{array}\right],
\end{aligned}
$$

where $T_{0}=0.25$ is the sampled period, $x(t)=\left[x_{1}(t), x_{2}(t)\right]^{T}$ is the state, $x_{1}(t)$ and $x_{2}(t)$ are the position and velocity of target at time $t T_{0} \cdot w(t)$, and $v_{i}(t)$ are independent Gaussian white noises with zero mean and unknown actual variances $\bar{Q}$ and $\bar{R}_{i}$, respectively. Taking the conservative noise variances $Q$ and $R_{i}$ satisfies $\bar{Q} \leq Q$ and $\bar{R}_{i} \leq R_{i}$. In the simulation, we take $Q=1, R_{1}=0.8, R_{2}=\operatorname{diag}(8,0.36), R_{3}=0.64, \bar{Q}=0.8$, $\bar{R}_{1}=0.65, \bar{R}_{2}=\operatorname{diag}(6,0.25)$, and $\bar{R}_{3}=0.54$.

Applying the local and fused robust steady-state Kalman filters, the actual and conservative filtering error variances are obtained in Table 1.

Table 1 verifies the accuracy relations (83)-(87), and it is easy to be verified that $P_{\mathrm{CI}} \leq P_{\mathrm{CI}}^{*}$ is satisfied. The traces of the error variances of the local and fused Kalman filters are compared in Table 2, which verify the accuracy relations (88)-(93).

In order to give a geometric interpretation of the matrix accuracy relations, the covariance ellipse is defined as the locus of points $\left\{x: x^{T} P^{-1} x=c\right\}$, where $P$ is the variance matrix and $c$ is a constant. Generally, we select $c=1$. It has been proved [32] that $P_{1} \leq P_{2}$ is equivalent to the covariance ellipse form $P_{1}$ by contains that form by $P_{2}$.

From Figures 1 and 2 , we see that the ellipse of $P^{(j)}(j=$ $0,1,2)$ is enclosed in these of $P_{m}, P_{d}, P_{s}$, and $P_{i}(i=1,2,3)$ which verifies the matrix accuracy relations (85)-(87). From Figure 3, we see that the ellipses of $\bar{P}^{(j)}(j=0,1,2)$ or $\bar{P}_{\theta}(\theta=1,2,3, m, s, d, \mathrm{CI})$ are enclosed in these of the 
TABLE 2: The accuracy comparison of local and fused robust Kalman filters.

\begin{tabular}{lcccccc}
\hline $\operatorname{tr} P_{1}$ & $\operatorname{tr} P_{2}$ & $\operatorname{tr} P_{3}$ & $\operatorname{tr} P_{m}$ & $\operatorname{tr} P_{d}$ & $\operatorname{tr} P_{s}$ & $\operatorname{tr} P_{\mathrm{CI}}^{*}, \operatorname{tr} P_{\mathrm{CI}}$ \\
\hline 0.5538 & 0.5245 & 0.4952 & 0.1942 & 0.2212 & 0.2725 & $0.4022,0.2360$ \\
\hline $\operatorname{tr} \bar{P}_{1}$ & $\operatorname{tr} \bar{P}_{2}$ & $\operatorname{tr} \bar{P}_{3}$ & $\operatorname{tr} \bar{P}_{m}$ & $\operatorname{tr} \bar{P}_{d}$ & $\operatorname{tr} \bar{P}_{s}$ & $\operatorname{tr} \bar{P}_{\mathrm{CI}}$ \\
\hline 0.4465 & 0.3815 & 0.4069 & 0.1485 & 0.1711 & 0.2131 & 0.1817 \\
\hline $\operatorname{tr} P^{(0)}$ & $\operatorname{tr} \bar{P}^{(0)}$ & $\operatorname{tr} P^{(1)}=\operatorname{tr} P^{(2)}$ & $\operatorname{tr} \bar{P}^{(1)}=\operatorname{tr} \bar{P}^{(2)}$ & & & \\
\hline 0.1795 & 0.1367 & 0.1795 & 0.1367 & & \\
\hline
\end{tabular}

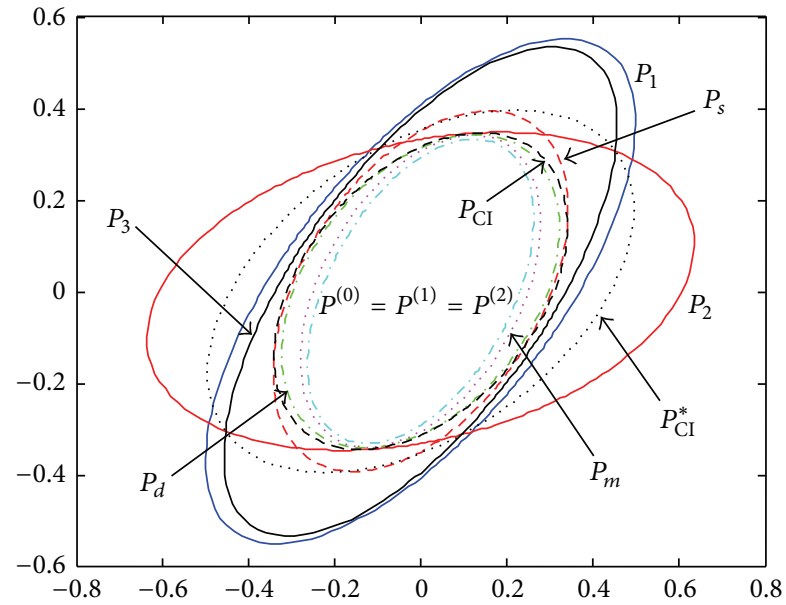

Figure 1: The ellipses of the conservative upper bounds of actual filtering error variances.

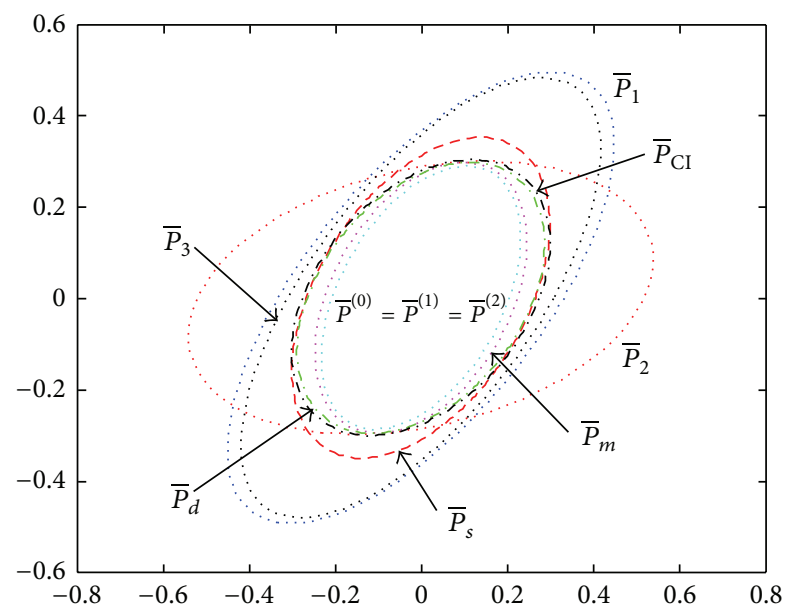

FIGURE 2: The ellipses of the actual filtering error variances.

conservative upper bound $P^{(j)}$ or $P_{\theta}$, respectively, which verify the robustness (83)-(87).

In order to verify the above theoretical accuracy relations, taking $\rho=200$ Monte-Carlo simulation runs, the mean square error (MSE) value at time $t$ of local or fused Kalman filters is defined as

$$
\begin{gathered}
\operatorname{MSE}_{\theta}(t)=\frac{1}{\rho} \sum_{k=1}^{\rho}\left(x^{(k)}(t)-\widehat{x}_{\theta}^{(k)}(t \mid t)\right)^{T} \\
\times\left(x^{(k)}(t)-\widehat{x}_{\theta}^{(k)}(t \mid t)\right), \\
\theta=1,2,3, m, s, d, \mathrm{CI},
\end{gathered}
$$

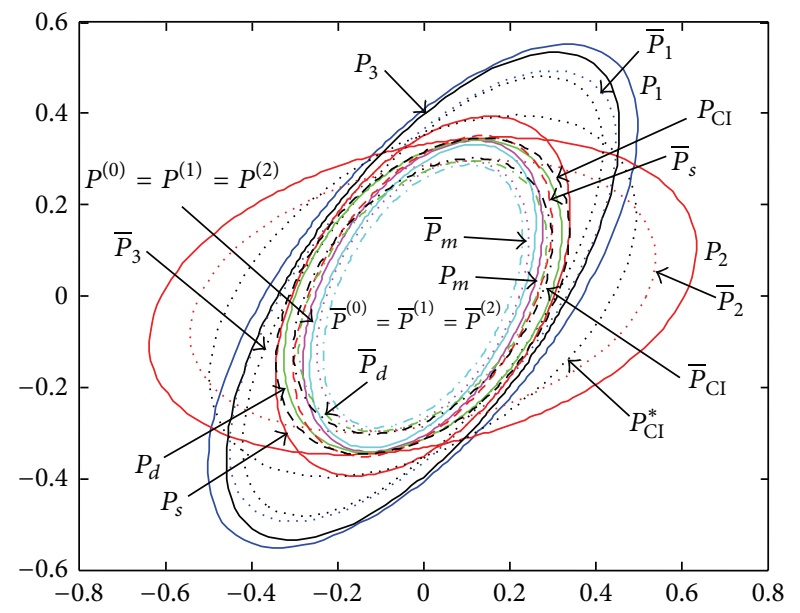

FIgURE 3: The ellipses of the actual and conservative filtering error variances.

$$
\begin{aligned}
\operatorname{MSE}_{j}(t)=\frac{1}{\rho} \sum_{k=1}^{\rho}\left(x^{(k)}(t)-\widehat{x}^{(k)(j)}(t \mid t)\right)^{T} & \\
& \times\left(x^{(k)}(t)-\widehat{x}^{(k)(j)}(t \mid t)\right), \quad j=0,1,2,
\end{aligned}
$$

where $x^{(k)}(t)$ or $\hat{x}_{\theta}^{(k)}(t \mid t)$ denotes the $k$ th realization of $x(t)$ or $\hat{x}_{\theta}(t \mid t)$ and $\hat{x}^{(k)(0)}(t \mid t)$ denotes the $k$ th realization of the centralized fuser. $t=1, \ldots, t_{f}$ are the recursive steps, the final step is $t_{f}=500$, and the MSE curves of the local and weighted fusion robust Kalman filters are shown in Figure 4, where the straight lines denote the traces of the theoretical error variances, respectively, the curves denote the values of the $\operatorname{MSE}_{\theta}(t)$ or $\operatorname{MSE}_{j}(t)$, and $\operatorname{tr} P^{(0)}=\operatorname{tr} P^{(1)}=\operatorname{tr} P^{(2)}$, $\operatorname{tr} \bar{P}^{(0)}=\operatorname{tr} \bar{P}^{(1)}=\operatorname{tr} \bar{P}^{(2)}$.

According to the consistency of the sampled variance, we have

$$
\begin{gathered}
\operatorname{MSE}_{\theta}(t) \longrightarrow \operatorname{tr} \bar{P}_{\theta}, \quad \text { as } t \longrightarrow \infty, \\
\rho \longrightarrow \infty, \quad(\theta=1,2,3, m, s, d, \mathrm{CI}), \\
\operatorname{MSE}_{j}(t) \longrightarrow \operatorname{tr} \bar{P}^{(j)}, \quad \text { as } t \longrightarrow \infty, \\
\rho \longrightarrow \infty, \quad(j=0,1,2) .
\end{gathered}
$$

From Figure 4 , we see that when $t$ is sufficiently large, the values of $\operatorname{MSE}_{i}(t)(i=\theta, j)$ are close to the corresponding 


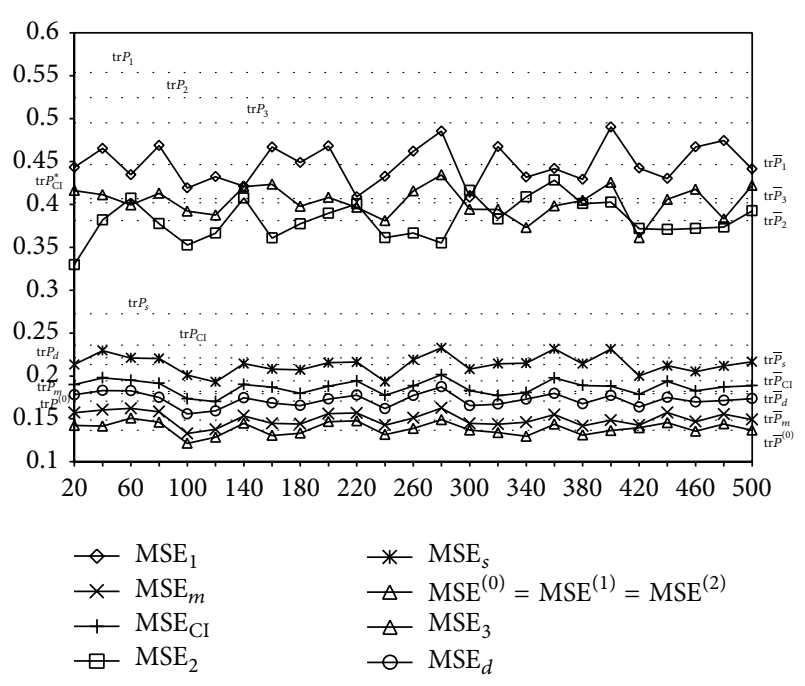

FIGURE 4: The MSE curves for local and fused robust Kalman filters.

theoretical values $\operatorname{tr} \bar{P}_{i}$, which verifies the consistency (97). We also see that $\operatorname{MSE}_{i}(t) \leq \operatorname{tr} P_{i}$, which verifies the accuracy relations (88) and (89).

\section{Conclusions}

For the multisensor time-invariant uncertain systems with uncertainties of noise variances, according to the minimax robust estimation principle, based on the worst-case conservative system with conservative upper bound of noise variances, using the ULMV optimal estimation rule and the steady-state Kalman filtering theory, the six robust weighted fusion steady-state Kalman filters have been presented. Their robustness was proved by using the Lyapunov equation method and their robust accuracy relations were proved. Compared with the method and results in [24], the main new contributions are as follows.

(1) Reference [24] presented an indirect design method for obtaining the robust weighted fusion steady-state Kalman filters which are obtained by taking the limit operations for the proposed time-varying robust Kalman fusers. This paper presented a simple direct design method based on the steady-state Kalman filtering theory, which can directly obtain the same steady-state results in [24], and avoided finding the time-varying robust Kalman fusers.

(2) A modified CI fusion method has been presented. A minimal upper bound of actual CI fusion error variances was presented based on the cross-covariance information. It reduces the conservativeness of the original upper bound without the cross-covariance information, and it improves and increases the robust accuracy of the CI fuser as shown in Remark 15. The ellipsoidal intersection (EI) fuser [33] with the crosscovariance information was developed and extended.

(3) Two robust weighted measurement fusion algorithms and the robust centralized fusion algorithm have been presented in a unified framework, their equivalence was proved, and they have the highest robust accuracy than the above other fusers. Only one weighted measurement fusion algorithm was presented in [24].

This paper is limited to the multisensor systems with uncertain noise variances; the extension of the proposed results to the multisensor systems with both the uncertain model parameters and noise variances is in the investigation.

\section{Conflict of Interests}

The authors declare that there is no conflict of interests regarding the publication of this paper.

\section{Acknowledgments}

This work is supported by the Natural Science Foundation of China under Grant NSFC-60874063 and Innovation and Scientific Research Foundation of Graduate Student of Heilongjiang Province under Grant YJSCX2012-263HLJ. The authors thank the reviewers and editors for their helpful and constructive comments, which are very valuable for improving quality of the paper.

\section{References}

[1] D. L. Hall and J. Llinas, "An introduction to multisensor data fusion," Proceedings of the IEEE, vol. 85, no. 1, pp. 6-23, 1997.

[2] X. R. Li, Y. Zhu, J. Wang, and C. Han, "Optimal linear estimation fusion-part I: unified fusion rules," IEEE Transactions on Information Theory, vol. 49, no. 9, pp. 2192-2323, 2003.

[3] Z.-L. Deng, Y. Gao, L. Mao, Y. Li, and G. Hao, "New approach to information fusion steady-state Kalman filtering," Automatica, vol. 41, no. 10, pp. 1695-1707, 2005.

[4] S.-L. Sun and Z.-L. Deng, "Multi-sensor optimal information fusion Kalman filter," Automatica, vol. 40, no. 6, pp. 1017-1023, 2004.

[5] Z. L. Deng, P. Zhang, W. J. Qi, Y. Gao, and J. F. Liu, “The accuracy comparison of multisensor covariance intersection fuser and three weighting fusers," Information Fusion, vol. 14, pp. 177-185, 2013.

[6] Q. Gan and C. J. Harris, "Comparison of two measurement fusion method for Kalman filter based multisensor data fusion," IEEE Transactions on Aerospace and Electronic Systems, vol. 37, no. 1, pp. 273-280, 2001.

[7] Y. Gao, C.-J. Ran, X.-J. Sun, and Z.-L. Deng, "Optimal and selftuning weighted measurement fusion Kalman filters and their asymptotic global optimality," International Journal of Adaptive Control and Signal Processing, vol. 24, no. 11, pp. 982-1004, 2010.

[8] C. J. Ran, Y. S. Hui, L. Gu, and Z. L. Deng, "Correlated measurement fusion steady-state Kalman filtering algorithms and their optimality," Acta Automatica Sinica, vol. 34, no. 3, pp. 233-239, 2008.

[9] Y. Theodor and U. Sharked, "Robust discrete-time minimumvariance filtering," IEEE Transactions on Signal Processing, vol. 44, no. 2, pp. 181-189, 1996.

[10] X. Zhu, Y. C. Soh, and L. Xie, "Design and analysis of discretetime robust Kalman filters," Automatica, vol. 38, no. 6, pp. 10691077, 2002. 
[11] X. Lu, L. Xie, H. Zhang, and W. Wang, "Robust Kalman filtering for discrete-time systems with measurement delay," IEEE Transactions on Circuits and Systems II: Express Briefs, vol. 54, no. 6, pp. 522-526, 2007.

[12] K. Xiong, C. L. Wei, and L. D. Liu, "Robust Kalman filtering for discrete-time nonlinear systems with parameter uncertainties," Aerospace Science and Technology, vol. 18, no. 1, pp. 15-24, 2012.

[13] F. Yang, Z. Wang, and Y. S. Hung, "Robust Kalman filtering for discrete time-varying uncertain systems with multiplicative noises," IEEE Transactions on Automatic Control, vol. 47, no. 7, pp. 1179-1183, 2002.

[14] M. S. Mahmoud, L. Xie, and Y. C. Soh, "Robust Kalman filtering for discrete state-delay systems," IEE Proceedings: Control Theory and Applications, vol. 147, no. 6, pp. 613-618, 2000.

[15] Y. K. Foo and Y. C. Soh, "Robust Kalman filtering for uncertain discrete-time systems with probabilistic parameters bounded within a polytope," Systems \& Control Letters, vol. 57, no. 6, pp. 482-488, 2008.

[16] L. Xie, L. Lu, D. Zhang, and H. Zhang, "Improved robust $H_{2}$ and $H_{\infty}$ filtering for uncertain discrete-time systems," Automatica, vol. 40, no. 5, pp. 873-880, 2004.

[17] F. Wang and V. Balakrishnan, "Robust steady-state filtering for systems with deterministic and stochastic uncertainties," IEEE Transactions on Signal Processing, vol. 51, no. 10, pp. 2550-2558, 2003.

[18] F. Yang and Y. Li, "Robust set-membership filtering for systems with missing measurement: a linear matrix inequality approach," IET Signal Processing, vol. 6, no. 4, pp. 341-347, 2012.

[19] X.-B. Jin, J. Bao, and J.-L. Zhang, "Centralized fusion estimation for uncertain multisensor system based on LMI method," in Proceedings of the IEEE International Conference on Mechatronics and Automation (ICMA '09), pp. 2383-2387, Changchun, China, August 2009.

[20] X. Qu and J. Zhou, "The optimal robust finite-horizon Kalman filtering for multiple sensors with different stochastic failure rates," Applied Mathematics Letters, vol. 26, no. 1, pp. 80-86, 2013.

[21] H. Xi, "The guaranteed estimation performance filter for discrete-time descriptor systems with uncertain noise," International Journal of Systems Science, vol. 28, no. 1, pp. 113-121, 1997.

[22] Z. Dong and Z. You, "Finite-horizon robust Kalman filtering for uncertain discrete time-varying systems with uncertaincovariance white noises," IEEE Signal Processing Letters, vol. 13, no. 8, pp. 493-496, 2006.

[23] L. Shi, K. H. Johansson, and R. M. Murray, "Kalman filtering with uncertain process and measurement noise covariances with application to state estimation in sensor networks," in Proceedings of the 16th IEEE International Conference on Control Applications (CCA '07), pp. 1031-1036, Singapore, October 2007.

[24] W. J. Qi, P. Zhang, and Z. L. Deng, "Robust weighted fusion Kalman filters for multisensor time-varying systems with uncertain noise variances," Signal Processing, vol. 99, pp. 185200, 2014.

[25] B. Chen, L. Yu, W.-A. Zhang, and A. Liu, "Robust information fusion estimator for multiple delay-tolerant sensors with different failure rates," IEEE Transactions on Circuits and Systems. I. Regular Papers, vol. 60, no. 2, pp. 401-414, 2013.

[26] J. Feng, Z. Wang, and M. Zeng, "Distributed weighted robust Kalman filter fusion for uncertain systems with autocorrelated and cross-correlated noises," Information Fusion, vol. 14, pp. 7886, 2013.
[27] A. Ahmad, M. Gani, and F. Yang, "Decentralized robust Kalman filtering for uncertain stochastic systems over heterogeneous sensor networks," Signal Processing, vol. 88, no. 8, pp. 1919-1928, 2008.

[28] S. J. Julier and J. K. Uhlmann, "Non-divergent estimation algorithm in the presence of unknown correlations," in Proceedings of the American Control Conference, vol. 4, pp. 2369-2373, June 1997.

[29] S. J. Julier and J. K. Uhlmann, "Simultaneous localization and map building using split covariance intersection," in Proceedings of the IEEE International Conference on Intelligent Robots and Systems, pp. 1257-1262, 2001.

[30] S. J. Julier and J. K. Uhlmann, "General decentralized data fusion with covariance intersection," in Handbook of Multisensor Data Fusion, M. E. Liggins, D. L. Hall, and J. Llinas, Eds., Theory and Practice, pp. 319-342, CRC Press, 2nd edition, 2009.

[31] W. Niehsen and R. B. Gmbh, "Information fusion based on fast covariance intersection filtering," in Proceedings of the 5th International Conference on Information Fusion, pp. 901-905, 2002.

[32] Z. Deng, P. Zhang, W. Qi, J. Liu, and Y. Gao, "Sequential covariance intersection fusion Kalman filter," Information Sciences, vol. 189, pp. 293-309, 2012.

[33] J. Sijs and M. Lazar, "State fusion with unknown correlation: ellipsoidal intersection," Automatica, vol. 48, no. 8, pp. 18741878, 2012.

[34] Q. Guo, S. Chen, H. Leung, and S. Liu, "Covariance intersection based image fusion technique with application to pansharpening in remote sensing," Information Sciences, vol. 180, no. 18, pp. 3434-3443, 2010.

[35] S. J. Julier and J. K. Uhlmann, "Using covarianceintersection for SLAM," Robotics and Autonomous Systems, vol. 55, no. 1, pp. 320, 2007.

[36] J. Cesar Bolzani de Campos Ferreira and J. Waldmann, "Covariance intersection-based sensor fusion for sounding rocket tracking and impact area prediction," Control Engineering Practice, vol. 15, no. 4, pp. 389-409, 2007.

[37] S. B. Lazarus, I. Ashokaraj, A. Tsourdos et al., "Vehicle localization using sensors data fusion via integration of covariance intersection and interval analysis," IEEE Sensors Journal, vol. 7, no. 9, pp. 1302-1314, 2007.

[38] T. Kailath, A. H. Sayed, and B. Hassibi, Linear Estimation, Prentice Hall, New York, NY, USA, 2000.

[39] E. W. Kamen and J. K. Su, Introduction to Optimal Estimation, Springer, London, UK, 1999.

[40] X. Qu, J. Zhou, E. Song, and Y. Zhu, "Minimax robust optimal estimation fusion in distributed multisensor systems with uncertainties," IEEE Signal Processing Letters, vol. 17, no. 9, pp. 811-814, 2010. 


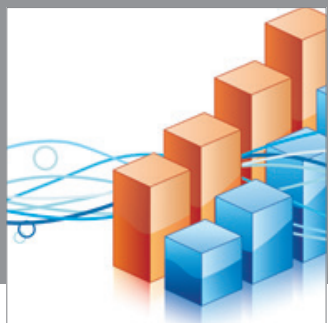

Advances in

Operations Research

mansans

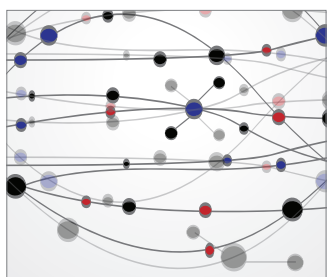

The Scientific World Journal
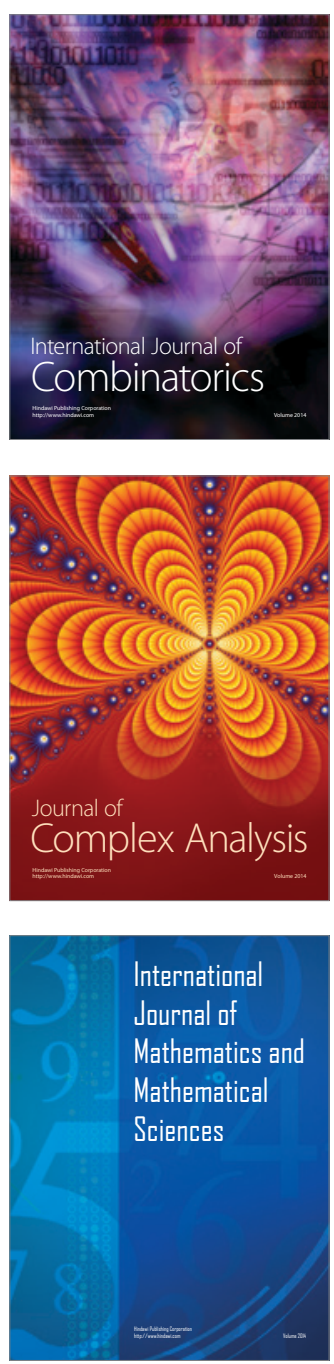
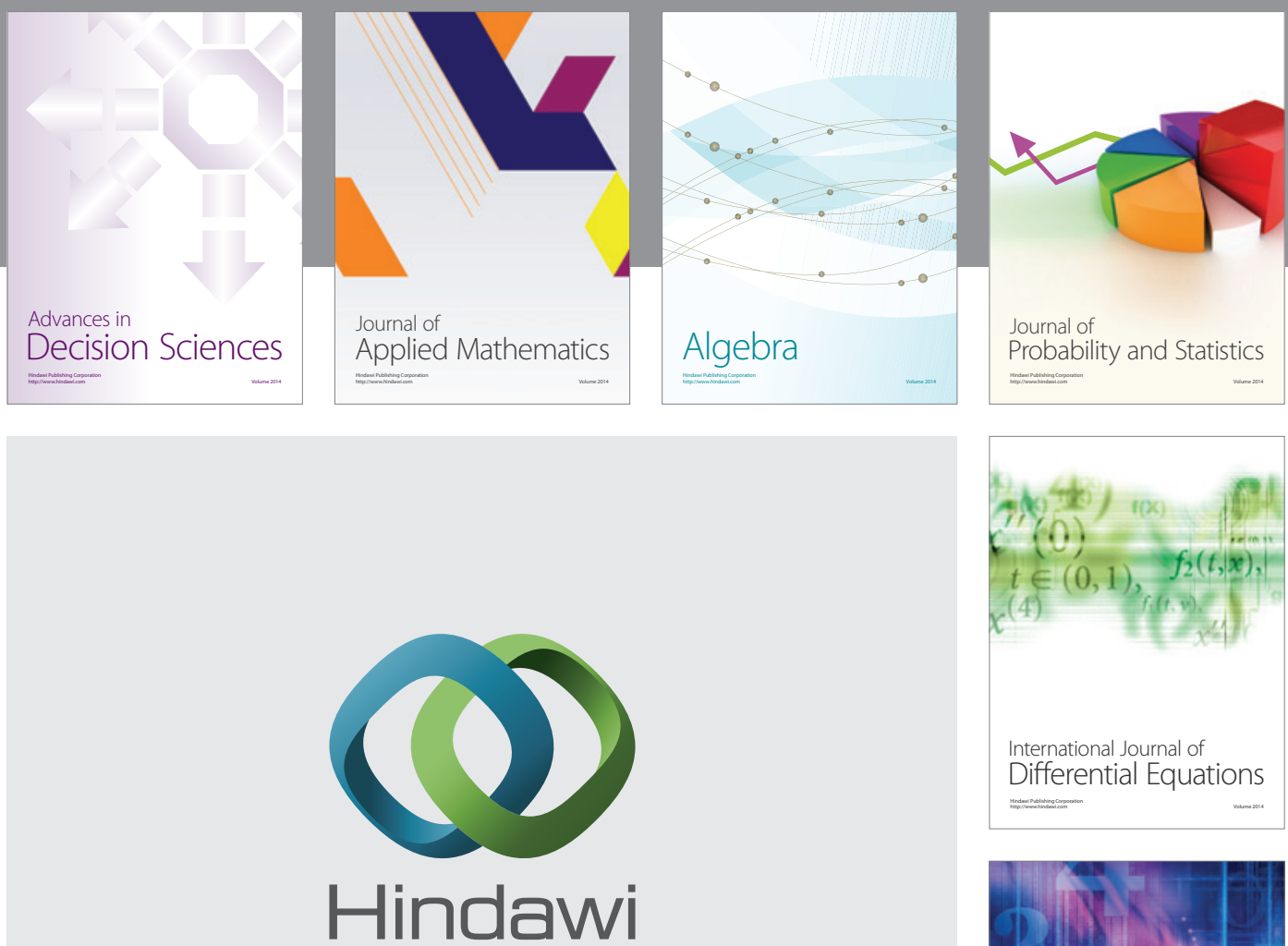

Submit your manuscripts at http://www.hindawi.com
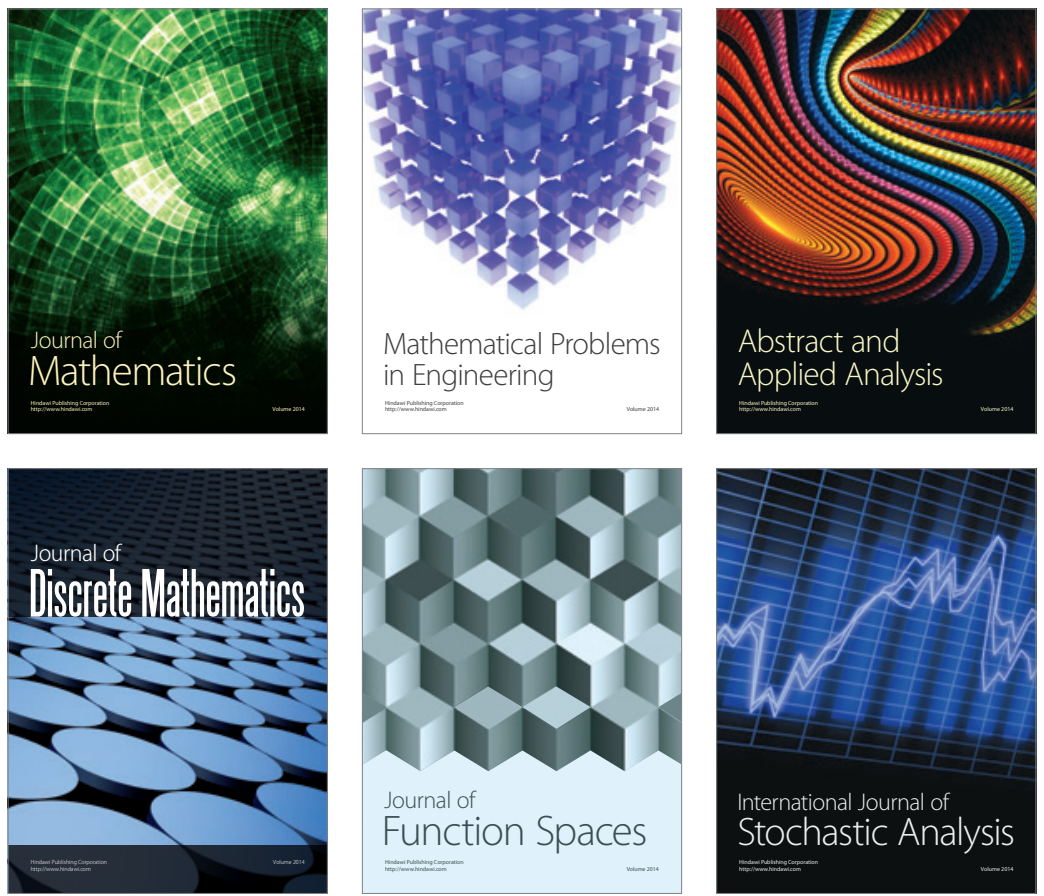

Journal of

Function Spaces

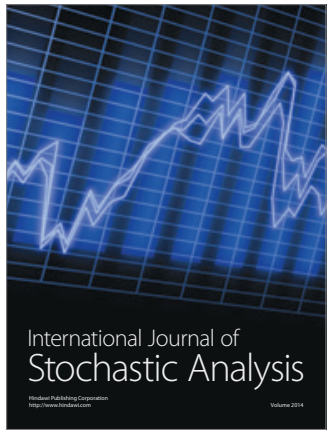

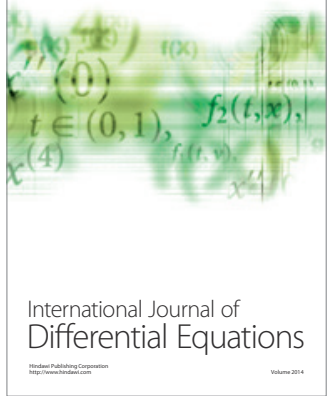
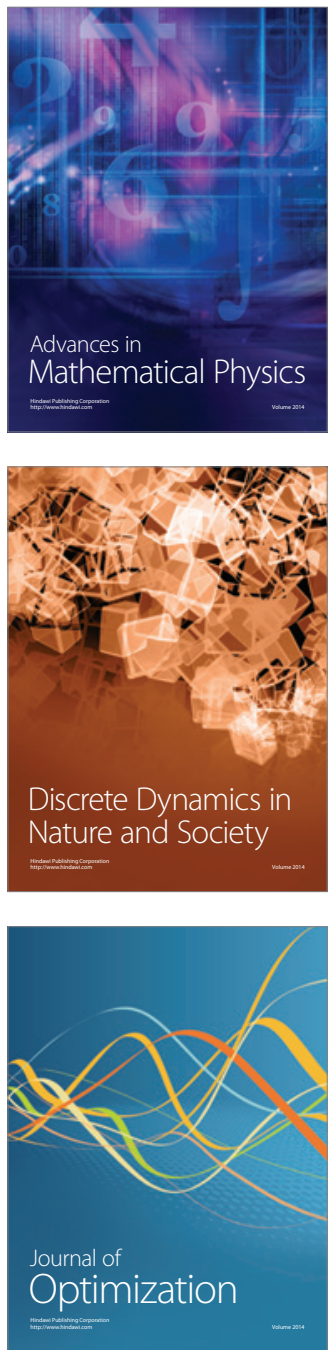